\title{
BEAM IMPACT STUDIES ON ILC COLLIMATORS
}

\author{
George Ellwood, STFC/RAL, Chilton, Didcot, Oxon, UK
}

Juan Luis Fernandez-Hernando, James Jones, STFC/DL/ASTeC, UK

Nigel Watson, Mark Slater, Birmingham University, Birmingham, UK

\begin{abstract}
Spoilers in the ILC Beam Delivery System are required to survive without failure a minimum of 1-2 direct impacts of $250 \mathrm{GeV}-500 \mathrm{GeV}$ bunch of electrons or positrons, in addition to maintaining low geometric and resistive wall wake fields. Simulations were completed to determine the energy deposition of an ILC bunch to a set of different spoiler designs. These shower simulations were used as inputs to thermal and mechanical studies using ANSYS. This paper presents the results of testing carried out at the Accelerator Test Facility at KEK used to validate the simulations. Results from the first phase of testing, in which electron bunches of varying charge were incident on TI-6Al-4V foils, are presented and compared with simulations.
\end{abstract}

\section{EXPERIMENTAL AIMS}

This experiment was part of a larger research programme that was tasked with designing the collimation system for the ILC. The overall aim of this area of research was to measure shock waves in a sample of Ti-6Al-4V alloy caused by rapid heating by the ATF beam using a VISAR to measure surface velocity of the sample. As this was a complex task, it was broken down into two phases. The first phase was to gain experience of operating at ATF, whilst the second phase was to carry out the instrumented test to measure shock waves.

The first phase is reported here. The aims of this test were first to gain experience working at ATF. The second was to commission the manipulator system that would be used in the second phase of testing. The third aim was to test aligning the sample with the electron beam. The fourth aim was to cause damage in the Ti-6Al-4V alloy sample; this would validate the FLUKA simulations.

\section{EXPERIMENTAL PROCEDURE}

The experiment used a location known colloquially as the 'ODR' location at ATF. The main equipment used was a vacuum manipulator that used stepper motors to control its motion. A sample of Ti-6Al-4V $100 \mu \mathrm{m}$ thick was placed in a sample holder. The sample holder could be manipulated within the beam line remotely.

One of the aims of the experiment was to align the sample with the electron beam. To do this a low charge beam was used. This beam was too low to cause damage. The sample would then be stepped down into the beam. When the beam was in contact with the bottom edge of the sample this would generate photons in a similar manner to a wire scanner. The downstream photon detector would then read out confirming the vertical position. The sample would then be moved horizontally with respect to the beam. The photon detector would continue to readout until the beam reached the edge of the sample. This would align the corner of the sample with the beam, and give the home location. A schematic of this is shown in figure 1.

Starting from the home location the sample could then be moved a set distance in $\mathrm{x}$ and $\mathrm{y}$ to find a sample test location, due to the precision of the stepper motor controlled manipulator, this sample test location could be found in post processing by measuring from the home location.

The beam charge could be increased and the beam size could be tightly focussed, this could give an energy density that should damage the sample. Different beam sizes and charges could then be tested. These should give different sized damage regions. As the location of each sample test location was known, and the beam conditions used at each location were recorded, the damage region could then be correlated to the beam conditions. This would allow for comparison with the FLUKA simulations.

\section{PREDICTIONS}

FLUKA was used to predict the amount of energy deposited in the sample by a beam strike. To do this several variables were estimated. The beam charge was estimated to be $1.28 \mathrm{GeV}$ with $2 \mathrm{E} 10$ charged particles per bunch. The beam size was estimated to be $1.9 \mu \mathrm{m} \times 0.5 \mu \mathrm{m}$ and another scenario of $20 \mu \times 2 \mu \mathrm{m}$ was simulated. The choice of beam size is critical. In order to create damage a small beam size is needed as this gives a high energy density. However in order to be able to measure the damage in post processing using a Scanning Electron Microscope a larger damage region is desired so that the damage is obvious when compared to the surrounding region. The predicted damage regions are shown in table 1 .

Table 1: Damage region predictions

\begin{tabular}{llll}
\hline $\begin{array}{l}\text { Bunch } \\
\left(\begin{array}{l}\sigma_{\mathrm{x}} \times \\
(\mu \mathrm{m})\end{array}\right.\end{array}$ & $\begin{array}{r}\text { size } \\
\left.\sigma_{\mathrm{y}}\right)\end{array}$ & $\begin{array}{l}\text { Estimated } \\
\text { damage region, } \\
x(\mu \mathrm{m})\end{array}$ & $\begin{array}{l}\text { Estimated damage } \\
\text { region, } y(\mu \mathrm{m})\end{array}$ \\
\hline $1.9 \times 0.5$ & 11 & 4 \\
\hline $20 \times 2$ & 45 & 5 \\
\hline
\end{tabular}



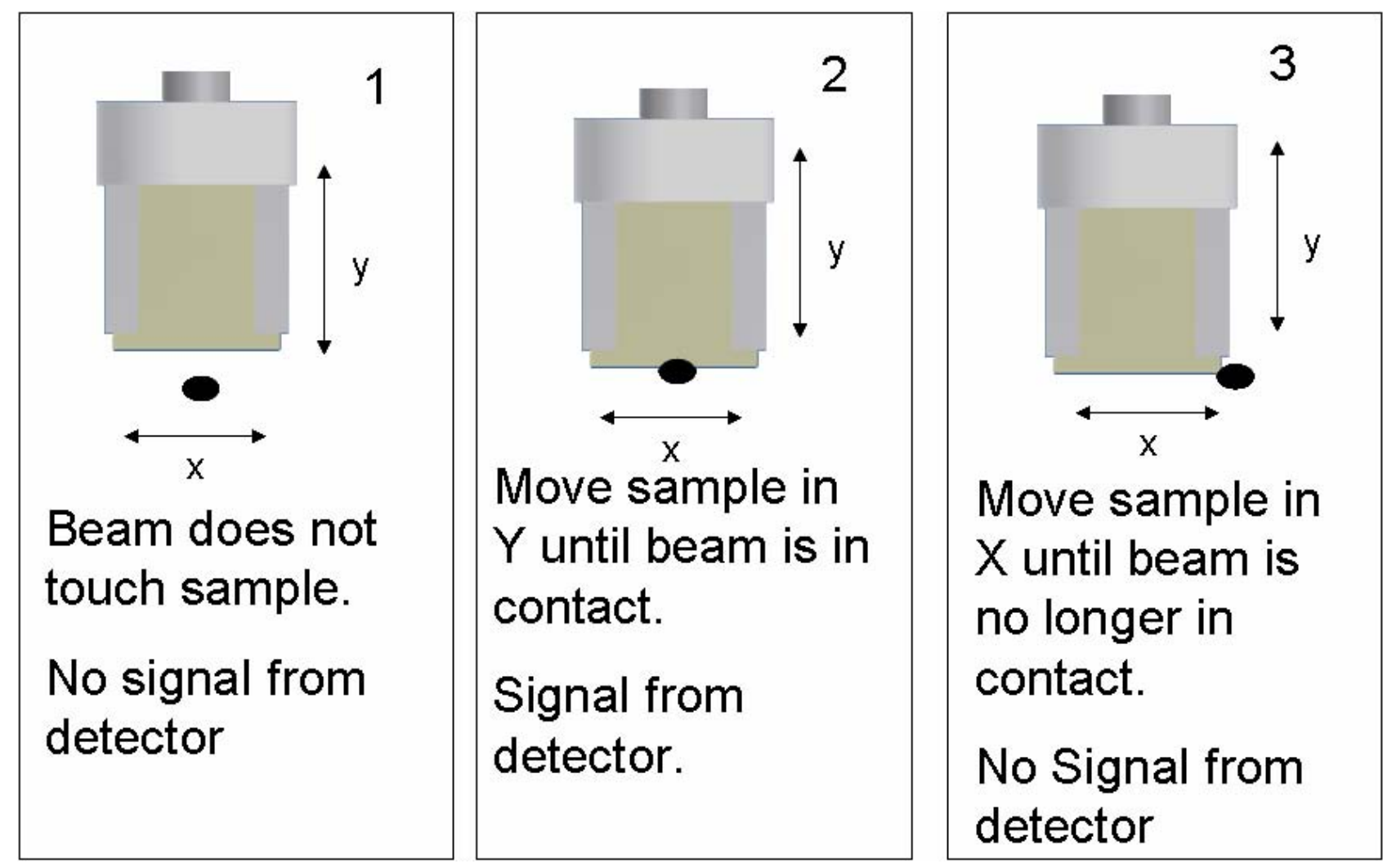

Figure 1: Schematic showing the alignment of the sample with the electron beam.

\section{RESULTS}

Whilst operating at ATF it was not possible to get the beam size or charge desired to cause damage to the sample. The smallest beam achieved was estimated to be $10 \mu \mathrm{m} \times 5 \mu \mathrm{m}$ at the ODR location. It was not possible to measure the beam size at this location because the wire scanner that is usually present at this location was removed to allow installation of the test equipment. The beam charge was also lower than assumed; the highest beam charge achieved was 1.2 E10 charged particles per bunch. According to the simulations this would not be enough to damage the sample.

Although the simulations predicted that the sample was unlikely to be damaged the test continued. This consisted of creating a pattern of beam strikes on the sample so that in post processing any damage caused to the sample would be systematic and obvious when viewed. The pattern of beam strikes is shown in figure 2 .

After testing the sample was inspected using a Scanning Electron Microscope. Areas of possible damage were located by using the method described in the experimental procedure, the home location was found and the areas were located in relation to this location.

Figure 3 shows a typical SEM image. This frame shows a region where there were several beam strikes; however there were no visible signs of beam damage. There are some imperfections in the sample surface however these are likely to be due to fabrication method used to create the sample. These imperfections were also seen in regions where there had not been a beam strike.

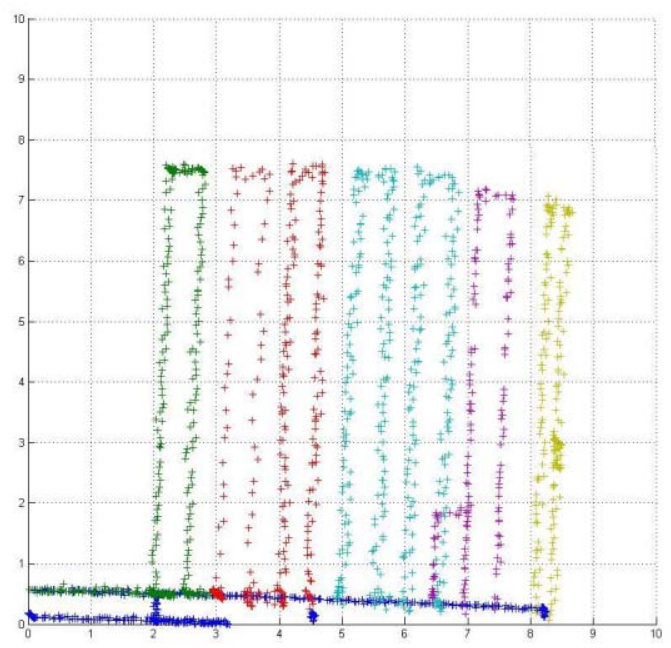

Figure 2: Schematic showing the pattern of beam strikes on the target. 


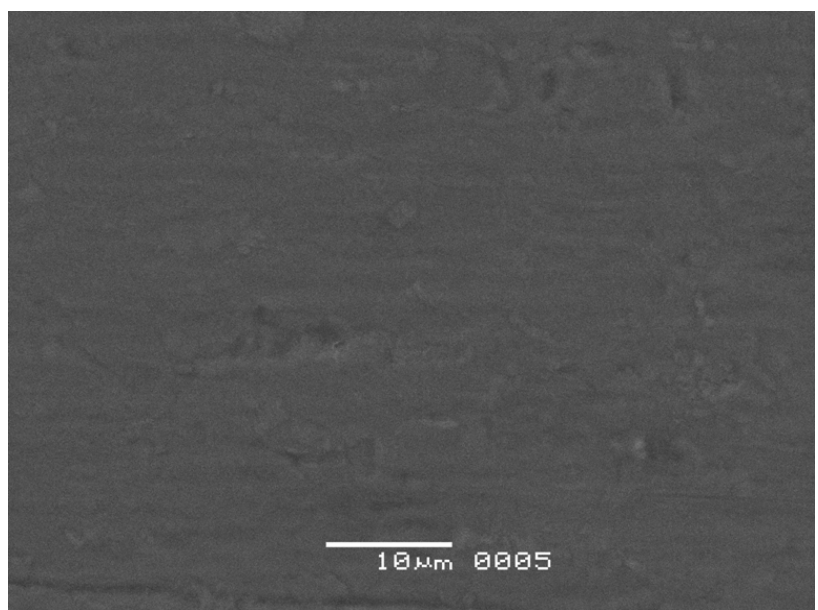

Figure 3: SEM image showing a beam strike region but without any signs of damage.

\section{CONCLUSIONS}

The beam test was largely successful. Of the four main aims three were accomplished. The team gained experience of working at ATF. This will help greatly when planning the second phase of the test. The test equipment was commissioned successfully. This also allowed the team to use the equipment from the ATF control room which could not be simulated elsewhere. The test established that it is possible to align the beam and the sample by using the readout from a downstream photon detector.

The test did not manage to produce damage in the sample. This was because on this test run it was not possible to achieve the beam conditions required. However the experience gained will allow for more realistic simulations of the beam conditions for the second phase of testing. 